\title{
Wissenschaftliches Programm auf dem 2. Interprofessionellen Gesundheitskongress 2016 in Dresden
}

\author{
6. HeilberufeSCIENCE-Symposium
}

\section{J. Klewer}

Online publiziert: 17. Oktober 2015

(C) Springer-Verlag Wien 2015

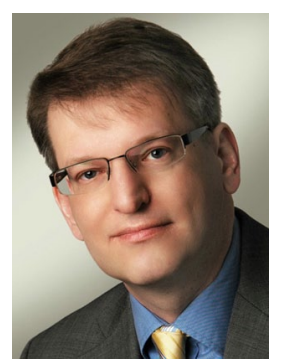

J. Klewer

Das erfolgreich verlaufene 5. HeilberufeSCIENCE-Symposium auf dem Interprofessionellen Gesundheitskongress in Dresden im April dieses Jahres war der Anlass, über eine Erweiterung des wissenschaftlichen Kongressprogramms nachzudenken. Außerdem bietet der neue Veranstaltungsort, das Maritim Congress Center Dresden direkt an der Elbe, sehr gut geeignete Räumlichkeiten. Wie in den Vorjahren wird das 6. HeilberufeSCIENCE-Symposium daher in bewährter Form den Kern des wissenschaftlichen Kongressteils des 2. Interprofessionellen Gesundheitskongresses am 8. und 9. April 2016 bilden. Das HeilberufeSCIENCE-Symposium wird um eine geführte Postersession erweitert, da nun ausreichend Platz für eine angemessene Präsentation der Poster vorhanden sein wird. Flankierend sollen einzelne Keynote Speaker und Diskussionsrunden das wissenschaftliche Programm ergänzen. Daher lade ich Sie herzlich ein, sich am 6. HeilberufeSCIENCE-Symposium mit einem Poster oder einem Vortrag zu beteiligen. Bitte reichen Sie Ihr Abstract bis zum 31. Januar 2016 ein. Weitere Informationen finden Sie im Call for Abstracts auf http://www.gesundheitskongresse.de.

J. Klewer $(\bowtie)$

Zwickau, Deutschland

E-Mail: joklewer@t-online.de
In dieser Ausgabe von HeilberufeSCIENCE bieten wir Ihnen 5 Beiträge, darunter 3 Übersichtsarbeiten. In einer Übersichtsarbeit haben sich Simon und Flaiz mithilfe einer Literaturrecherche im Deutschen Ärzteblatt mit den Sichtweisen der Ärzteschaft zur Professionalisierung der Pflege auseinandergesetzt. Ebenfalls anhand einer systematischen Literaturübersicht betrachten Rädel-Ablass und Scupin umfassend den Wandel der familialen Pflege. Halbmayr-Kubicsek arbeitet - wiederum über eine systematische Literaturrecherche - die Effekte von ,,advance care planning (ACP)“ in der stationären Altenpflege heraus. In einer Originalarbeit versuchen Hanel et al. mit einer empirischen Analyse von Bewerberdatensätzen einer gesetzlichen Krankenkasse Prädiktoren für den Ausbildungserfolge von Auszubildenden zu Sozialversicherungsfachangestellten zu ermitteln. Die wissenschaftliche Kurzmitteilung von Steinert et al. stellt die Ergebnisse einer Studie zum Selbst-Monitoring der physischen Aktivität von Senioren durch den Einsatz von Schrittzählern dar. Dabei zeigte sich, dass die tägliche Schrittzahl der Senioren durch das Selbst-Monitoring signifikant erhöht werden konnte.

Hiermit lade ich Sie wieder ein, die 5 wissenschaftlichen Arbeiten dieser Ausgabe von HeilberufeSCIENCE kritisch $\mathrm{zu}$ lesen und in den wissenschaftlichen Dialog einzutreten - und freue mich auf Ihr Abstract für das 6. HeilberufeSCIENCE-Symposium in Dresden.

Zum Abschluss wünsche ich Ihnen eine schöne Weihnachtszeit und alles Gute für das Jahr 2016!

Ihr

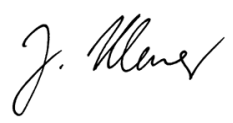

J. Klewer

Interessenkonflikt J. Klewer gibt an, dass kein Interessenkonflikt besteht. 